\title{
Error Augmentation: The Alternative Approach to Treat Brain Injury
}

Carmeli $\mathrm{E}^{*}$

Department of Physical Therapy, University of Haifa, Israel

*Corresponding author: Carmeli E, Associate Professor, Department of Physical Therapy, University of Haifa, Israel, Tel: 972-4-828-8397; E-mail: ecarmeli@univ.haifa.ac.il

Received date: November 30, 2016; Accepted date: December 19, 2016; Published date: December 22, 2016

Copyright: $\odot 2016$ Carmeli E. This is an open-access article distributed under the terms of the Creative Commons Attribution License, which permits unrestricted use, distribution, and reproduction in any medium, provided the original author and source are credited.

\begin{abstract}
Enhancement of sensory motor performance is vital in rehabilitation after brain injury. This short communication discusses a new approach in motor learning and rehabilitation: The error augmentation (EA) which utilizes incorrect visual and proprioceptive feedback to improve motor adaptation. In EA technology, the computer distinguishes and amplifies errors in a patient's movement from a preferred trajectory, or modifies the visual feedback of the movement trajectory, and consequently gives emphasis to visual and sensory feedback. The existence of this error in visual input requires from patients to reinforce their motor control as they work against the error-driven disturbance to the movements, at the same time it enhances motivation to learn by making even little errors seem great.
\end{abstract}

Keywords: Error augmentation; Aging brain; Stroke; Rehabilitation

\section{Short Communication}

Every year, numerous people worldwide sustain traumatic brain injuries, strokes and Parkinson's Disease, resulted in daily life activity impairments and dysfunction [1].

The restoration of optimal function is the utmost important aspect of becoming independence individual, yet arm-hand mobility remains highly challenged of rehabilitation goal [2], and beyond spontaneous recovery, the traditional or conventional therapies (e.g., Neuro Development Treatment, Proprioceptive Neuromuscular Facilitation, Constrain-induced, massed practice) yield relatively modest outcomes $[3,4]$.

Recently, there is a growing trend toward using feed back technology to rehabilitate the sensory-motor ability by reducing motor impairment more effectively than does conventional therapy of stroke and Traumatic Brain Injury survivors [5].

Motor learning is characterized by long-lasting changes in motor performance, indicating that retention has indeed taken place, and evidenced by normal movement patterns [6].

The most update approach for enhancing motor recovery includes a robotic interface known as error augmentation (EA) [7]. EA utilizes incorrect feedback to boost motor recovery after neurological damage. In EA technology, the computer distinguishes and amplifies errors in a patient's movement from a preferred trajectory, or modifies the visual feedback of the movement trajectory, and consequently gives emphasis to visual and sensory feedback.

The existence of this error in visual input requires from patients to reinforce their motor control as they work against the error-driven disturbance to the movements.

Recently, we have found that augmenting error indeed enhance motor learning [8].
The scientific explanation of such learning process is through creation of neural networks and central adaptation of motor skill acquisition [9].

Adaptation is a key element to understand motor learning. It allows us to determine whether the CNS is still capable to adjust to more patterns of movement. Yet, the durability of adaptation is noticeably differing among people with CNS damage [10].

The washout period does not demolish the memory created during adaptation, although it may temporally inhibits its expression, which returns unexpectedly after period of time.

Since inherent feedback mechanisms are frequently damaged in brain injury, providing better feedback by making errors more obvious to the senses, its notion to be valuable, in that a patient learns faster when the error is larger [11].

Moreover, bigger errors are expected to enhance motivation to learn by making even little errors seem great [12]. In addition, rising error can guide to larger signal-to-noise ratios for sensory feedback and selfevaluation. It is crucial to remember that EA empiric technology facilitates extremely accurate and truthful information, done automatically, and able to produce a wide range of forces and motions.

In summery, to deal with the question in regard to motor learning and adaptation that eventually leads to long-term retention of the motor task, EA is excellent approach. It includes gradual exposure to the perturbation forces, which makes the after-effect smaller when the perturbation forces are switched off, it implements repeated and massed training, and if patient repeatedly practice arm-hand movements even several times a day over 3-4 weeks, he can develop a new "learned" calibration for the context that initially drove adaptation [13]. In other words, he no longer has to adapt from one behavior to the other, but instead have two learned behaviors that he can switch between. Therefore, the training protocol must be longer and comprises additional treatments and more repetitions to enable the CNS to make lasting changes in movement patterns.

However, evaluating the neuro physiological process resulting from EA training may encourage researchers to keep investigating this clinical field, as many of the stroke's patients still do not improve arm 
Citation: Carmeli E (2016) Error Augmentation: The Alternative Approach to Treat Brain Injury. J Aging Sci 4: 165. doi:

Page 2 of 2

ability. Topics like the period of time the effect of treatment continues, and whether the change in motor performance accomplished by motor learning or by increased use of compensation strategies should be further established. Furthermore, it is not yet known whether adaptation process may lead to long lasting motor memory as happen in motor learning.

In conclusion, there is growing evidence supporting the use of EA to improve motor performance of the upper extremity for stroke's patients.

\section{References}

1. Kitago T, Goldsmith J, Harran M, Kane L, Berard J, et al. (2015) Robotic therapy for chronic stroke: general recovery of impairment or improved task-specific skill? J Neurophysiol 114: 1885-1894.

2. Krabben T, Prange GB, Molier BI, Stienen AH, Jannink MJ, et al. (2012) Influence of gravity compensation training on synergistic movement patterns of the upper extremity after stroke, a pilot study. J Neuroeng Rehabil 9: 44

3. Abdollahi F, Case Lazarro ED, Listenberger M, Kenyon RV, Kovic M, et al. (2014) Error augmentation enhancing arm recovery in individuals with chronic stroke: a randomized crossover design. Neurorehabil Neural Repair 28: 120-128.

4. Patton JL, Stoykov ME, Kovic M, Mussa-Ivaldi FA (2006) Evaluation of robotic training forces that either enhance or reduce error in chronic hemiparetic stroke survivors. Exp Brain Res 168: 368-383.

5. Klamroth-Marganska V, Blanco J, Campen K, Curt A, Dietz V, et al. (2014) Three-dimensional, task-specific robot therapy of the arm after stroke: a multicentre, parallel-group randomised trial. Lancet Neurol 3: 159-166.

6. Fluet GG, Patel J, Qiu Q, Yarossi M, Massood S, et al. (2016) Motor skill changes and neurophysiologic adaptation to recovery-oriented virtual rehabilitation of hand function in a person with subacute stroke: a case study. Disabil Rehabil 1-8.

7. Israely S, Carmeli E (2016) Error augmentation as a possible technique for improving upper extremity motor performance after a stroke - a systematic review. Top Stroke Rehabil 23: 116-125.

8. Givon-Mayo R, Simon E, Karpin H, Ori A, Israely S, et al. (2014) Error enhancement of the velocity component in the course of Stroke patients reaching movements. A preliminary study. Int J Ther Rehabil 21: 160-168.

9. Patton JL, Wei YJ, Bajaj P, Scheidt RA (2013) Visuomotor learning enhanced by augmenting instantaneous trajectory error feedback during reaching. PLoS One 8: e46466.

10. Luu TP, He Y, Brown S, Nakagame S, Contreras-Vidal JL (2016) Gait adaptation to visual kinematic perturbations using a real-time closedloop brain-computer interface to a virtual reality avatar. J Neural Eng 13: 036006.

11. Huang FC, Patton JL, Mussa-Ivaldi FA (2010) Manual skill generalization enhanced by negative viscosity. J Neurophysiol 104: 2008-2019.

12. Molier BI, Prange GB, Krabben T, Stienen A, van der Kooij H, et al. (2011) Effect of position feedback during task-oriented upper-limb training after stroke: Five-case pilot study. J Rehabil Res Dev 48: 1109-1118.

13. Bastian AJ (2008) Understanding sensorimotor adaptation and learning for ehabilitation. Curr Opin Neurol 21: 628-633. 